Pacific Journal of Mathematics

INVARIANT SUBMODULES OF UNIMODULAR HERMITIAN 


\title{
INVARIANT SUBMODULES OF UNIMODULAR HERMITIAN FORMS
}

\author{
D. G. JAMES
}

Let $M$ be a unimodular lattice on an indefinite hermitian space over an algebraic number field. The submodules of $M$ invariant under the action of the special unitary group of $M$ are classified. Generators for the local unitary groups of $M$ are also determined.

1. Introduction. Let $F$ be an algebraic number field of finite degree and $K$ a quadratic extension of $F$. Let $V$ be an indefinite hermitian space over $K$ of finite dimension $n \geqq 3$ and $\Phi: V \times V \rightarrow K$ the associated nondegenerate hermitian form on $V$ with respect to the nontrivial automorphism of $K$ over $F$. Assume $V$ supports a unimodular lattice $M$ (in the sense of 0 'Meara [7; $\S 82 \mathrm{G}]$ for quadratic spaces). Denote by $U(V)$ the unitary group of $V$ and by $U(M)$ the subgroup of isometries in $U(V)$ that leave $M$ invariant. We will classify the sublattices of $M$ that are invariant under the action of the special unitary group $S U(M)$. The problem is first solved locally; the global result is then obtained by applying the approximation theorem of Shimura $[8 ; 5.12]$.

We now consider localization (see also $[2 ; \S 2]$ and [8]). Let $\mathfrak{p}$ be a finite prime spot of $F$ and $F_{\mathfrak{p}}$ the corresponding local field. Put $K_{\mathfrak{p}}=K \otimes_{F} F_{\mathfrak{p}}$ and $V_{\mathfrak{p}}=V \otimes_{F} F_{\mathfrak{p}}$. Making the standard identifications, we have $K \subseteq K_{\mathfrak{p}}, F_{\mathfrak{p}} \subseteq K_{\mathfrak{p}}$ and $V \cong V_{\mathfrak{p}}$. The hermitian form $\Phi$ on $V$ extends naturally to an hermitian form on $V_{p}$. Let $\mathfrak{D}$ be the ring of integers in $F, \mathfrak{D}_{\mathfrak{p}}$ the (topological) closure of $\mathfrak{D}$ in $F_{\mathfrak{p}}$ and $\mathfrak{D}_{\mathfrak{p}}$ the integral closure of $\mathfrak{o}_{\mathfrak{p}}$ in $K_{\mathfrak{p}}$. Put $M_{\mathfrak{p}}=\mathfrak{O}_{\mathfrak{p}} M \cong V_{\mathfrak{p}}$. Locally, we must study the submodules of $M_{\mathfrak{p}}$ invariant under the action of $S U\left(M_{p}\right)$. Except when $K_{\mathfrak{p}}$ is a ramified extension of a dyadic field $F_{p}$, the classification will be trivial. For ramified dyadic extensions, it is necessary to determine a set of generators of $U\left(M_{\mathfrak{p}}\right)$ before the classification can be determined.

We now state the main results.

THEOREM A. Let $M$ be a unimodular lattice on an indefinite hermitian space of dimension $n \geqq 3$ over an algebraic number field. Then a sublattice $N$ of $M$ is invariant under the action of the special unitary group $S U(M)$ if and only if for all finite prime spots $\mathfrak{p}$ of $F$, the localization $N_{\mathfrak{p}}=\mathfrak{O}_{\mathfrak{p}} N$ is invariant under the ac- 
tion of $S U\left(M_{\mathfrak{p}}\right)$.

For $x$ in $V_{\mathfrak{p}}$, define $2 q(x)=\Phi(x, x)$, and let $M_{\mathfrak{p}^{*}}$ be the sublattice of $M_{\mathfrak{p}}$ generated by the $x$ in $M_{\mathfrak{p}}$ with $q(x)$ in $\mathfrak{o}_{\mathfrak{p}}$. Let

$$
M_{\mathfrak{p}}^{*}=\left\{x \in V_{\mathfrak{p}} \mid \Phi\left(x, M_{p^{*}}\right) \subseteq \mathfrak{O}_{\mathfrak{p}}\right\}
$$

be the dual lattice of $M_{\mathfrak{p}^{*}}$. Then $M_{\mathfrak{p}^{*}} \subseteq M_{\mathfrak{p}} \subseteq M_{\mathfrak{p}}^{*}$ and, except when $K_{\mathfrak{p}}$ is a ramified extension of a dyadic local field $F_{\mathfrak{p}}$, we will show later that $M_{p^{*}}=M_{\mathfrak{p}}^{*}$. A sublattice $N_{\mathfrak{p}}$ of $M_{\mathfrak{p}}^{*}$ is called primitive if $N_{\downarrow}$ is not contained in $\pi M_{p}^{*}$ for any prime element $\pi \in \mathfrak{N}_{p}$. Clearly,

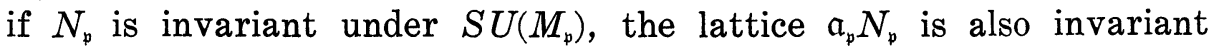
for any fractional ideal $\mathfrak{a}_{\mathfrak{p}}$ in $\mathfrak{O}_{\mathfrak{p}}$. It is therefore enough to classify locally the primitive invariant sublattices of $M_{\uparrow}^{*}$.

THEOREM B. A primitive sublattice $N_{p}$ of $M_{p}^{*}$ is invariant under the action of $S U\left(M_{\mathfrak{p}}\right)$ if and only if $M_{p^{*}} \subseteq N_{p}$, except when the following three conditions all apply:

(i ) $K_{\mathfrak{p}}$ is a totally ramified extension of the 2-adic field $\boldsymbol{Q}_{2}$,

(ii) $K_{\mathfrak{p}}$ is a ramified prime extension of $F_{\mathfrak{p}}$,

(iii) $\operatorname{dim} V_{p}=3$ or 4 .

In particular, except when $K_{\mathrm{p}}$ is a ramified extension of a dyadic field $F_{p}$, the only primitive invariant lattice is $M_{p}$.

Theorem B will be proven for the various cases in $\S \S 2-4$ and the exceptional 3 and 4 dimensional cases studied in $\S 5$. Theorem $\mathrm{A}$ is established in the final section. The special case where $F$ is the field of rational numbers is also studied in detail.

The approach here follows that given for quadratic spaces in [5] and [6].

2. Local isometries. In this and next three sections we are only concerned with local problems.

The structure of $\mathfrak{O}_{\mathfrak{p}}$ over $\mathfrak{o}_{\mathfrak{p}}$ depends on the prime $\mathfrak{p}$. If $\mathfrak{p}$ splits in $K$, then $K_{\mathfrak{p}}=F_{\mathfrak{p}} \times F_{\mathfrak{p}}$ and $\mathfrak{O}_{\mathfrak{p}}=\mathfrak{o}_{\mathfrak{p}} \times \mathfrak{o}_{\mathfrak{p}}$. In this case the involution ${ }^{*}$ on $K$ becomes $(\alpha, \beta)^{*}=(\beta, \alpha)$ on $K_{p}$. If $\mathfrak{p}$ does not split in $K$, we may take $K_{\mathfrak{p}}=F_{\mathfrak{p}}(\zeta)$ where $\zeta^{2} \in F_{\mathfrak{p}}$ and $\zeta^{*}=-\zeta$. Fix a prime $\pi$ in $K_{\mathfrak{p}}$ and $p$ in $F_{\mathfrak{p}}$ and let $e=\operatorname{ord}_{p} 2$. If $\mathfrak{p}$ is dyadic, there are now three possible types of extensions of $K_{p}$ over $F_{p}$; the details are an application of $[7 ; 63.2,63.3]$.

(i) $K_{\mathfrak{p}}$ is an unramified extension of $F_{p}$. Then $\zeta^{2}=1+4 \delta$ with $\delta$ a unit in $F_{\mathfrak{p}}$ and $\mathfrak{D}_{\mathfrak{p}}$ consists of all the elements $(\alpha+\zeta \beta) / 2$ with $\alpha, \beta \in \mathfrak{v}_{\mathfrak{p}}$ and $\alpha \equiv \beta \bmod 2 \mathfrak{o}_{p}$. 
(ii) $K_{p}$ is a ramified extension of $F_{p}$ and $\zeta$ is a prime in $K_{p}$ the ramified prime case. Now we may assume $\pi=\zeta, p=\pi \pi^{*}$ and $\mathfrak{O}_{\mathfrak{p}}$ is generated over $\mathfrak{D}_{\mathfrak{p}}$ by 1 and $\pi$.

(iii) $K_{p}$ is a ramified extension of $F_{\mathfrak{p}}$ and $\zeta$ is a unit in $K_{\mathfrak{p}}$ - the ramified unit case. We now have $\zeta^{2}=1+p^{2 h+1} \delta$ for some unit $\delta$ in $F_{\ddagger}$ and some rational integer $h$ with $0 \leqq h<e$. Put $\pi=(1+\zeta) p^{-h}$ so that $\pi \pi^{*}=-p \delta$. Here $\mathfrak{O}_{\mathfrak{p}}$ consists of the elements $(\alpha+\zeta \beta) p^{-h}$ with $\alpha, \beta \in \mathfrak{D}_{\mathfrak{p}}$ and $\alpha \equiv \beta \bmod p^{h} \mathfrak{D}_{\mathfrak{p}}$.

In the nondyadic (nonsplit) case $\mathfrak{D}_{p}$ is generated over $\mathfrak{o}_{\mathfrak{p}}$ by 1 and $\zeta$ provided we choose $\zeta$ to be a prime or a unit according as the extension is ramified or not.

Thus if $K_{\mathfrak{p}} / F_{\mathfrak{p}}$ is a quadratic extension of fields, $\mathfrak{N}_{\mathfrak{p}}$ consists of the elements $(\alpha+\zeta \beta) p^{-h}$ with $\alpha, \beta \in \mathfrak{o}_{\mathfrak{p}}$ and $\alpha \equiv \beta \bmod p^{h} \mathfrak{o}_{\mathfrak{p}}$, where we define $h=0$ in the nondyadic and ramified prime dyadic cases, and $h=e$ in the unramified dyadic case.

Since $M_{\mathfrak{p}}$ is a unimodular $\mathfrak{O}_{\mathfrak{p}}$-lattice with rank at least three, it is split by a hyperbolic plane (if $\mathfrak{p}$ splits in $K$ this can be easily verified, otherwise see [4; 7.1, 8.1a, 10.3]). Hence $M_{\mathfrak{p}}=H_{\mathfrak{p} \perp} L_{\mathfrak{p}}$ where $H_{\mathfrak{p}}=\mathfrak{O}_{\mathfrak{p}} u+\mathfrak{O}_{\mathfrak{p}} v$ is a hyperbolic plane with $q(u)=q(v)=0$ and $\Phi(u, v)=1$. This choice of $u$ and $v$ will be fixed throughout the local discussion.

We now describe the standard isometries in the unitary group $U\left(M_{p}\right)$ that are needed. The norm and trace mappings from $K_{p}$ to $F_{p}$ are denoted by $\mathscr{N}$ and $\mathscr{T}$, respectively, and our convention for the hermitian form $\Phi$ on $V_{\mathfrak{p}}$ is $\Phi(\alpha x, \beta y)=\alpha^{*} \Phi(x, y) \beta$.

Let $\lambda$ in $\mathfrak{O}_{\mathfrak{p}}$ have $\mathscr{T}(\lambda)=0$. The transvection $T_{\lambda}(u)$ is defined by

$$
T_{\lambda}(u)(z)=z+\lambda \Phi(u, z) u, \quad z \in M_{\mathfrak{p}} \text {. }
$$

Then $\operatorname{det} T_{\lambda}(u)=1$ so that $T_{\lambda}(u)$ is in $S U\left(M_{\mathfrak{p}}\right)$. Similarly, $T_{\lambda}(v) \in$ $S U\left(M_{p}\right)$.

Let $\lambda$ in $K_{\mathfrak{p}}$ satisfy $\mathscr{T}(\lambda)=2 \mathscr{N}(\lambda)$. For $x$ in $M_{\mathfrak{p}}$ with $\lambda q(x)^{-1}$ in $\mathfrak{S}_{\mathfrak{p}}$, define the symmetry $\Psi_{\lambda}(x)$ by

$$
\Psi_{\lambda}(x)(z)=z-\lambda \Phi(x, z) q(x)^{-1} x, \quad z \in M_{\mathfrak{p}} .
$$

Then $\operatorname{det} \Psi_{\lambda}(x)=1-2 \lambda$ and $\Psi_{\lambda}(x) \in U\left(M_{\mathfrak{p}}\right)$.

Recall that $M_{p^{*}}$ is the sublattice of $M_{\mathfrak{p}}$ generated by those $x$ in $M_{\mathfrak{p}}$ with $q(x) \in \mathfrak{D}_{\mathfrak{p}}$. Since $2 q(x)=\Phi(x, x)$, in the nondyadic case $M_{p^{*}}=$ $M_{p}$. This is also true when $\mathfrak{p}$ splits in $K$; for the involution on $K_{p}=F_{p} \times F_{p}$ is given by $(\alpha, \beta)^{*}=(\beta, \alpha)$, so that for $x$ in $M_{p}$,

$$
q((1,0) x)=\mathscr{N}(1,0) q(x)=0 .
$$

Thus $(1,0) x \in M_{\mathfrak{p}^{*}}$ and $x=(1,1) x$ is in $M_{\mathfrak{p}^{*}}$. 
Proposition 2.1. Let $F_{\mathfrak{p}}$ be a dyadic local field with $\mathfrak{p}$ not split in $K$. Then

$$
M_{p^{*}}=\left\{x \in M_{\mathfrak{p}} \mid p^{h} q(x) \in \mathfrak{D}_{\mathfrak{p}}\right\} .
$$

In particular, $M_{\mathfrak{p}^{*}}=M_{\mathfrak{p}}$ when $K_{\mathfrak{p}}$ is an unramified extension of $F_{\mathfrak{p}}$.

Proof. Let $S$ be the set of all elements $x$ in $M_{\mathfrak{p}}$ with $p^{h} q(x)$ in $\mathfrak{o}_{\mathfrak{p}}$. Since $\mathscr{T}\left(\mathfrak{D}_{\mathfrak{p}}\right) \cong 2 p^{-h} \mathfrak{o}_{\mathfrak{p}}$ and

$$
q(x+y)=q(x)+q(y)+\mathscr{T}(\Phi(x, y)) / 2,
$$

it follows that $S$ is an $\mathfrak{S}_{p}$-module. Hence $M_{p^{*}} \subseteq S$. We now prove the converse inclusion. For $x$ in $S$, let $x=y+z$ with $y \in H_{\mathfrak{p}}$ and $z \in L_{p}$. Clearly, $u, v$ and consequently $y$ are in $S$. Therefore, $z=$ $x-y$ is in $S$ and $p^{h} q(z) \in \mathfrak{D}_{p}$. Let $w=u-\alpha v+z$ where $\alpha=q(z)(1+\zeta)$ is in $\mathfrak{S}_{p}$. Then $q(w)=0$ and $w \in M_{p^{*}}$. Hence $z \in M_{p^{*}}$ and $S \subseteq M_{p^{*}}$, proving the proposition.

Fix $\mu$ in $\mathfrak{D}_{\mathfrak{p}}$ such that $\mathscr{T}(\mu)=2$. For $x$ in $L_{\mathfrak{p}}$ with $\mu q(x)$ in $\mathfrak{O}_{\mathfrak{p}}$, define the Siegel transformation $E(u, x)$ by

$$
E(u, x)(z)=z-\Phi(u, z) x+\Phi(x, z) u-\mu q(x) \Phi(u, z) u .
$$

Then $\operatorname{det} E(u, x)=1$ and $E(u, x)$ is in $S U\left(M_{\uparrow}\right)$. Similarly, define $E(v, x)$. Fix $\mu=1$ except when $F_{p}$ is dyadic and $K_{p}$ is either an unramified or a ramified unit extension of $F_{p}$. In these exceptional cases fix $\mu=1+\zeta \in p^{h} \mathfrak{D}_{p}$. Except for the split dyadic case, it is now sufficient to choose $x$ in $L_{\mathfrak{p}} \cap M_{\mathfrak{p}^{*}}$ for $E(u, x)$ to be an integral isometry. Let $\mathscr{E}$ be the subgroup of $S U\left(M_{\mathfrak{p}}\right)$ generated by the Siegel transformations.

In the following three sections we classify locally the primitive sublattices of $M_{p}^{*}$ invariant under the action of the special unitary group $S U\left(M_{\mathfrak{p}}\right)$. We conclude this section with three observations. Assume that $\mathfrak{p}$ does not split in $K$.

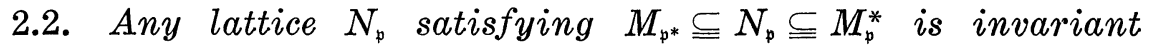
under the action of $\mathscr{E}$.

Proof. Let $z \in N_{p}$ and $x \in L_{p} \cap M_{p^{*}}$. Then $\Phi(x, z) \in \mathfrak{O}_{\mathfrak{p}}$ and

$$
E(u, x)(z) \equiv z \bmod M_{\mathfrak{p}^{*}} \text {. }
$$

Hence $E(u, x)(z)$ and, likewise, $E(v, x)(z)$ lies in $N_{p}$. The result follows.

2.3. If $N_{\mathfrak{p}}$ is invariant under $S U\left(M_{\mathfrak{p}}\right)$ and $u \in N_{\mathfrak{p}}$ or $v \in N_{\mathfrak{p}}$, then $M_{p^{*}} \subseteq N_{\mathfrak{p}}$.

Proof. For any $x$ in $L_{\mathfrak{p}}$ with $q(x)^{-1}$ in $\mathfrak{O}_{\mathfrak{p}}$, we have $\Psi_{1}(u-v) \Psi_{1}(x)$ 
is in $S U\left(M_{\mathfrak{p}}\right)$. This isometry interchanges $u$ and $v$, so that $H_{\mathfrak{p}} \subseteq N_{\mathfrak{p}}$. Let $y \in L_{\mathfrak{p}} \cap M_{\mathfrak{p}^{*}}$. Then $E(u, y)(v)$ is in $N_{\mathfrak{p}}$ and hence $y \in N_{\mathfrak{p}}$. Thus $M_{\mathfrak{p}^{*}} \subseteq N_{\mathfrak{p}}$.

2.4. Assume either $K_{\mathfrak{p}}$ is an unramified extension of $F_{\mathfrak{p}}$ or $F_{\mathfrak{p}}$ is a nondyadic field. Then $M_{\mathfrak{p}}$ is the unique primitive sublattice invariant under the action of $S U\left(M_{p}\right)$.

Proof. Let $N_{\downarrow}$ be a primitive invariant sublattice. It suffices by 2.3 to show that $u \in N_{p}$, since under our assumptions $M_{p^{*}}=M_{p}$. Since $N_{p} \nsubseteq \pi M_{p}$, there exists $z$ in $N_{\mathfrak{p}}$ with $z \notin \pi M_{p}$. Let $z=\alpha u+$ $\beta v+t$ where $t \in L_{p}$. If $\alpha$ and $\beta$ are nonunits, there exists $r$ in $L_{p}$ such that $\Phi(r, t)=1$ (since $z \notin \pi M_{p}^{*}$ ). The coefficient of $v$ in $E(v, r)(z) \in$

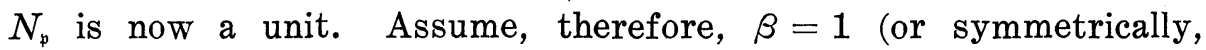
$\alpha=1$ ). If $K_{\mathfrak{p}}=F_{\downarrow}(\zeta)$ is an unramified extension of $F_{p}, \zeta$ is a unit. Then $T_{\zeta}(u)(z)=z+\zeta u$ is in $N_{\mathfrak{p}}$. Hence $u \in N_{\mathfrak{p}}$ and the result follows. Now assume $F_{p}$ is a nondyadic field. Then $E(u, t)(z)=\gamma u+v$ is in $N_{\mathfrak{p}}$ for some $\gamma$ in $\mathfrak{N}_{p}$. Let $w \in L_{p}$ have $q(w)$ a unit. Applying $E(u, \rho w)$ to $\gamma u+v \in N_{\mathfrak{p}}$ with $\rho=1,-1$ gives $\rho w+q(w) u$ is in $N_{p}$. Since 2 is now a unit, it follows that $u$ is in $N_{p}$ and hence $N_{p}=M_{p}$.

Theorem $B$ has now been established except when either $\mathfrak{p}$ splits in $K$, or $K_{\mathfrak{p}}$ is a ramified extension of a dyadic field $F_{p}$.

3. Split extensions. Assume $\mathfrak{p}$ splits in $K$ so that $K_{\mathfrak{p}}=F_{\mathfrak{p}} \times F_{\mathfrak{p}}$ and $\mathfrak{O}_{\mathfrak{p}}=\mathfrak{o}_{\mathfrak{p}} \times \mathfrak{o}_{\mathfrak{p}}$. Let $N_{\mathfrak{p}}$ be a primitive invariant sublattice of $M_{\mathfrak{p}}^{*}=M_{\mathfrak{p}}=H_{\mathfrak{p}} \perp L_{\mathfrak{p}}$. We wish to prove $N_{\mathfrak{p}}=M_{\mathfrak{p}}$. Since $N_{\mathfrak{p}} \nsubseteq \pi M_{\mathfrak{p}}$ for any prime element $\pi$ in $\mathfrak{O}_{\mathfrak{p}}$, there exists $x \in N_{\mathfrak{p}}$ with $x \notin \pi M_{\mathfrak{p}}$. Let $x=\alpha u+\beta v+t$ with $t \in L_{p}$. If $\beta$ (or $\alpha$ ) is a unit in $\mathfrak{O}_{p}$, we may assume $\beta=1$. Then, since $\mathscr{T}(1,-1)=0$, it follows that

$$
T_{(1,-1)}(u)(x)=x+(1,-1) u
$$

is in $N_{p}$. Thus $(1,-1) u$ and $u$ are in $N_{p}$. As in 2.3, we now have $H_{p} \subseteq N_{p}$. Let $y \in L_{p}$. Then $E(u,(1,0) y)(v)$ is in $N_{p}$. Hence $(1,0) y$, and likewise $(0,1) y$, are in $N_{p}$. Consequently, $y \in N_{p}$ and $N_{p}=M_{p}$.

Now assume that neither $\alpha=\left(\alpha_{1}, \alpha_{2}\right)$ nor $\beta=\left(\beta_{1}, \beta_{2}\right)$ is a unit. If $\alpha_{1}$ is a unit in $\mathfrak{o}_{p}$, replacing $x$ by $T_{(1,-1)}(v)(x)$ if necessary, we may assume $\beta_{1}$ is also a unit. Hence, unless both $\alpha_{1}$ and $\beta_{1}$ are nonunits, or both $\alpha_{2}$ and $\beta_{2}$ are nonunits, we arrange that $\beta$ becomes a unit in $\mathfrak{O}_{p}$ and we are finished. Assume, therefore, $\alpha_{1}, \beta_{1} \in p 0_{p}$. Since $x \notin \pi M_{p}$, there exists $y$ in $M_{\mathfrak{p}}$ such that $\Phi(x, y)=(1,1)$. Hence, there exists $r \in L_{\mathfrak{p}}$ such that $\Phi(t, r)=(?, 1)$. In $E(u,(0,1) r)(x)$ the new coefficient of $u$ has first component a unit. The second component is unchanged. We can thus arrange that $\beta$ becomes a unit in $\mathfrak{N}_{\mathfrak{p}}$, and consequently $N_{\mathfrak{p}}=M_{\mathfrak{p}}$. 
4. Ramified dyadic extensions. Now let $K_{\mathfrak{p}}$ be a ramified extension of the dyadic field $F_{p}$. Before classifying the primitive invariant sublattices in this case it is necessary to determine a set of generators for $U\left(M_{\mathfrak{p}}\right)$. Special cases have already appeared in the work of Baeza [1] and Hayakawa [3], but it appears better to modify the approach in [5].

By $[4 ; 10.3]$, we can split hyperbolic planes and write

$$
M_{\mathfrak{p}}=H_{\mathfrak{p}} \perp J_{\mathfrak{p}} \perp B_{\mathfrak{p}}
$$

where $J_{\mathfrak{p}}$ is an orthogonal sum of hyperbolic planes and rank $B_{\mathfrak{p}} \leqq 2$. Then $J_{p}$ has dual bases $w_{1}, \cdots, w_{m}$ and $z_{1}, \cdots, z_{m}$ such that $\Phi\left(w_{i}, z_{j}\right)=$ $\delta_{i j}, 1 \leqq i, j \leqq m$. Recall that $\mathscr{E}$ is the subgroup of $S U\left(M_{\mathfrak{p}}\right)$ generated by the Siegel transformations defined in $\S 2$.

\section{Proposition 4.1. $U\left(M_{\mathfrak{p}}\right)$ is generated by $\mathscr{E}$ and $U\left(H_{\mathfrak{p}} \perp B_{\mathfrak{p}}\right)$.}

Proof. Let $\varphi \in U\left(M_{p}\right)$. We reduce $\varphi$ to the identity using the given isometries. Let $w_{1}, \cdots, w_{m}$ and $z_{1}, \cdots, z_{m}$ be dual bases of $J_{p}$, as above, and assume for some $k \leqq m$ that $\varphi\left(w_{j}\right)=w_{j}, 1 \leqq j \leqq$ $k-1$ (at worst, $k=1$ ). Let

$$
\varphi\left(u+w_{k}\right)=\varepsilon u+\beta v+t
$$

where $t \in J_{p} \perp B_{p}$. We want $\varepsilon$ to be a unit. Assume $\varepsilon$ is not a unit. If $\beta$ is a unit, use the isometry in $U\left(H_{p}\right)$ which interchanges $u$ and $v$. If $\beta$ is not a unit, let $\varphi\left(z_{k}\right)$ have component $r$ in $J_{p} \perp B_{p}$. Then $\Phi(t, r)$ is a unit. Since $z_{k} \in M_{p^{*}}$, it follows that $r \in M_{p^{*}}$. Also, $\Phi\left(r, w_{j}\right)=\Phi\left(\varphi\left(z_{k}\right), \varphi\left(w_{j}\right)\right)=0$ for $1 \leqq j \leqq k-1$. Now replace $\varphi$ by $E(u, r) \varphi$ and the new coefficient of $u$ is a unit.

We may now assume $\varepsilon$ is a unit. Let $s=t-w_{k}$. Then

$$
\Phi\left(s, w_{j}\right)=\Phi\left(\phi\left(u+w_{k}\right)-w_{k}, w_{j}\right)=0
$$

for $1 \leqq j \leqq k-1$. Also, since $q(t) \equiv q\left(w_{k}\right) \bmod p^{-h} \mathfrak{o}_{\mathfrak{p}}$, we have $s \in M_{p^{*}}$. Put

$$
\psi^{\prime}=E\left(u,-\varepsilon^{*} z_{k}\right) T_{\lambda}(v) E\left(v, \varepsilon^{-1} s\right) \varphi E\left(u, z_{k}\right)
$$

where $\lambda \in \mathfrak{D}_{\mathfrak{p}}$ is to be chosen subject to the restraint $\mathscr{T}(\lambda)=0$. Then $\psi\left(w_{j}\right)=w_{j}$ for $1 \leqq j \leqq k-1$. Choose $\lambda$ such that

$$
E\left(v, \varepsilon^{-1} s\right) \varphi E\left(u, z_{k}\right)\left(w_{k}\right)=\varepsilon(u-\lambda v)+w_{k} .
$$

Then $\mathscr{T}(\lambda)=0$ and $\psi\left(w_{k}\right)=w_{k}$. If $\psi$ is generated by the given isometries, so is $\varphi$. The result now follows by induction on $k$.

This proposition reduces the question of generators for $U\left(M_{\mathfrak{p}}\right)$ to 
the cases rank $M_{\mathfrak{p}}=3,4$. It can be easily verified that $U\left(H_{\mathfrak{p}}\right)$ is generated by symmetries and transvections. Also, if rank $B_{\mathfrak{p}}=2$ the basis $w, z$ of $B_{p}$ can be chosen such that $\Phi(w, z)=1$ and $z \in M_{p^{*}}$ (see $[4 ; 9.2]$ ).

THEOREM 4.2. $U\left(M_{\mathfrak{p}}\right)$ is generated by $\mathscr{E}, U\left(H_{\mathfrak{p}}\right)$ and symmetries on $B_{p}$.

Proof. We need only consider rank $M_{\mathfrak{p}}=3,4$.

(i) Let rank $M_{\mathfrak{p}}=4$ and $M_{\mathfrak{p}}=H_{\mathfrak{p}} \perp B_{\mathfrak{p}}$ with $B_{\mathfrak{p}}$ having a basis as above. We reduce $\varphi$ in $U\left(M_{\mathfrak{p}}\right)$ to the identity using the given isometries. From the proof of Proposition 4.1, we may assume $\varphi(w)=w$. In fact, if $w \in M_{p^{*}}$, the proposition proves the theorem. Now assume $w \notin M_{p^{*}}$. Put $r=w-2 q(w) z$ so that $\Phi(r, w)=0$. Then

$$
\varphi(z)=\alpha u+\beta v+z+\gamma r
$$

for some $\alpha, \beta$ in $\mathfrak{D}_{\mathfrak{p}}$ and $\gamma$ in $\pi \mathfrak{O}_{\mathfrak{p}}\left(\gamma r \in M_{\mathfrak{p}^{*}}\right)$. Let

$$
\mathscr{H}_{z}=\{x \in M \mid \Phi(x, z)=1\}=w+H_{\mathfrak{p}} \perp \mathfrak{D}_{\mathfrak{p}}(z-2 q(z) w)
$$

be the characteristic set of $z$ (cf. [5; p. 429]). Then

$$
q\left(\mathscr{C l}_{\varphi(z)}\right)=q\left(\mathscr{C}_{z}\right) \equiv q(w) \bmod p^{-h} \mathfrak{o}_{\mathfrak{p}} .
$$

Since $\left(1-\alpha^{*}\right) w+v$ is in $\mathscr{C}_{\varphi(z)}$, it follows that $q(\alpha w) \in p^{-h} \mathfrak{D}_{\mathfrak{p}}$ and hence $\alpha w \in M_{\mathfrak{p}^{*}}$. Similarly, $\beta w \in M_{p^{*}}$. Interchanging $u$ and $v$ if necessary, we have $\beta=\alpha \lambda$ with $\lambda=\left(\lambda_{1}+\lambda_{2} \zeta\right) p^{-h}$ in $\mathfrak{O}_{\mathfrak{p}}$ and $\lambda_{1} \equiv \lambda_{2} \bmod p^{h}$. Using a transvection, we can then arrange that $\lambda \in \mathfrak{D}_{\mathfrak{p}}$ in the ramified prime case and $\lambda \in \pi 0_{p}$ in the ramified unit case. In the ramified prime case the proof can be completed by modifying the argument in $\left[5 ; 2.4\right.$ ]; the symmetry on $B_{p}$ needed is $\Psi_{\delta}(r)$ with $\delta \in \mathcal{O}_{p}$. In the ramified unit case we proceed as follows. The coefficient of $v$ in $E(v, \xi r) \varphi(z)$ is zero if

$$
\alpha \lambda+\xi^{*} \Phi(r, z+\gamma r)=\mu q(\xi r) \alpha .
$$

Here $\mu=1+\zeta=\pi p^{h}$ and $\varepsilon=\Phi(r, z+\gamma r)$ is a unit. By Hensel's lemma there exists a root $\xi$ of the form $\xi=\varepsilon \pi^{*} \alpha^{*} \rho$ with $\rho$ in $\mathrm{o}_{p}$. Similarly, the coefficient of $u$ can be made zero and we may assume $\varphi(z)=z+\gamma r$. Put $\delta=\gamma q(w)=-\gamma q(r) \Phi(z, r)^{-1}$. Then $\mathscr{T}(\delta)=2 \mathscr{N}(\delta)$ and $\Psi_{\delta}(r)^{-1} \varphi$ acts as the identity on both $w$ and $z$. This completes the proof in this case.

(ii) Let rank $M_{\mathfrak{p}}=3$ and $M_{\mathfrak{p}}=H_{\mathfrak{p}} \perp \mathfrak{O}_{\mathfrak{p}} w$ where $2 q(w)$ is a unit. Again, we can reduce $\varphi$ in $U\left(M_{p}\right)$ to the identity by the isometries. Let

$$
\varphi(w)=\alpha(u+\lambda v)+\eta w
$$


where $\eta$ is a unit. Moreover, as in the previous case, we may assume $\lambda$ is in $\pi \mathfrak{b}_{\mathfrak{p}}$ (resp. $\mathfrak{b}_{\mathfrak{p}}$ ) in the ramified unit (resp. prime) case. Since

$$
q\left(\mathfrak{D}_{\mathfrak{p}} \varphi(w)^{\perp}\right)=q\left(\mathfrak{D}_{\mathfrak{p}} w^{\perp}\right)=q\left(H_{\mathfrak{p}}\right) \leqq p^{-h_{\mathfrak{D}}},
$$

it follows that $\alpha w \in M_{p^{*}}$. Using Siegel transformations we can reduce to the case $\varphi(w)=\varepsilon w$, although in the ramified prime case it is necessary to use the fact that $\mathscr{N}(\eta) \equiv 1 \bmod 4$ and hence $\mathscr{N}(\eta)$ is a square. Finally, since $\mathscr{N}(\varepsilon)=1$, putting $\delta=(1-\varepsilon) / 2$ gives $\mathscr{T}(\delta)=2 \mathscr{N}(\delta)$ and $\Psi_{\delta}(w)^{-1} \varphi$ fixes $w$. This completes the proof.

COROLLARY 4.3. Except in the ramified unit case with the rank of $M_{\mathfrak{p}}$ even, all lattices $N_{\mathfrak{p}}$ satisfying

$$
M_{\mathfrak{p}^{*}} \subseteq N_{\mathfrak{p}} \subseteq M_{\mathfrak{p}}^{*}
$$

are invariant under the action of $U\left(M_{p}\right)$.

Proof. This follows from 2.2 and the easily verified fact that $U\left(H_{p}\right)$ and the symmetries used in the proof of the theorem preserve such $N_{p}$.

COROLlaRY 4.4. In the ramified unit case with rank $M_{\mathfrak{p}}$ even, all lattices between $M_{p^{*}}$ and $M_{\mathfrak{p}}^{*}$ are $S U\left(M_{\mathfrak{p}}\right)$-invariant.

Proof. Symmetries $\Psi_{\delta}$ in $U\left(H_{p}\right)$ have $p^{h} \delta \in \mathcal{S}_{p}$ and $\operatorname{det} \Psi_{\delta} \equiv$ $1 \bmod 2 p^{-h}$. Hence, for $\phi$ in $S U\left(M_{\mathfrak{p}}\right)$ in the proof of Theorem 2.2, the only symmetries $\Psi_{\hat{\delta}}(r)$ on $B_{\mathfrak{p}}$ needed will also have $p^{h} \delta \in \mathfrak{N}_{p}$. These symmetries leave invariant lattices between $M_{p^{*}}$ and $M_{p}^{*}$.

We now investigate the converse. Let $N_{p}$ be a primitive $S U\left(M_{p}\right)$-invariant sublattice of $M_{p}^{*}$. As in 2.4, there exists $x=\alpha u+$ $v+t$ in $N_{\mathfrak{p}}$ with $t \in L_{\mathfrak{p}}^{*}$ (letting $M_{\mathfrak{p}}^{*}=H_{\mathfrak{p}} \perp L_{\mathfrak{p}}^{*}$ ). In the ramified unit case $\zeta$ is a unit and $\mathscr{T}(\zeta)=0$. Since $T_{\zeta}(u)(x) \in N_{\mathfrak{p}}$, it follows that $\zeta u \in N_{\mathfrak{p}}$. By 2.3, $M_{\mathfrak{p}^{*}} \subseteq N_{\mathfrak{p}}$, completing the proof of Theorem B in this case. Finally, the ramified prime case. If $\operatorname{dim} V_{\mathfrak{p}} \geqq 5$, then $L_{\mathfrak{p}}$ is split by a hyperbolic plane $H_{\mathfrak{p}}^{\prime}=\mathfrak{S}_{\mathfrak{p}} u^{\prime}+\mathfrak{O}_{\mathfrak{p}} v^{\prime}$. Applying $E\left(u, u^{\prime}\right)$ to $x$, we obtain $u^{\prime}-\Phi\left(u^{\prime}, t\right) u$ is in $N_{p}$. Applying $E\left(u, v^{\prime}\right)$ now gives $u \in N_{p}$ and hence $M_{p^{*}} \subseteq N_{p}$. Assume, therefore, the rank of $M_{p}$ is 3

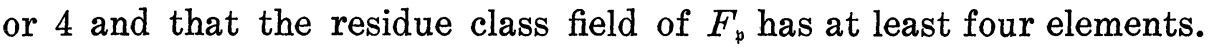
Let $\varepsilon$ be a unit in $F_{\mathfrak{p}}$ with $\varepsilon^{2} \not \equiv 1 \bmod p$. The proof of Theorem B is now easily completed by using the isometry $u \mapsto \varepsilon u, v \mapsto \varepsilon^{-1} v$ on $x$ to obtain $v \in N_{p}$. The exceptional case is studied in the next section.

5. Exceptional invariant lattices. In this section $F_{q}$ is a totally ramified extension of the 2-adic field $\boldsymbol{Q}_{2}$ and $K_{\mathfrak{p}}$ is a ramified prime 
extension of $F_{p}$. Thus the residue class fields of both $F_{\mathfrak{p}}$ and $K_{\mathfrak{p}}$ have only two elements.

We consider first the case with $\operatorname{dim} V_{\mathfrak{p}}=3$ so that $M_{\mathfrak{p}}=H_{\mathfrak{p}} \perp \mathfrak{O}_{\mathfrak{p}} w$. Then $M_{\mathfrak{p}^{*}}=H_{\mathfrak{p}} \perp \mathfrak{N}_{\mathfrak{p}} \pi^{e} w$ and $M_{\mathfrak{p}}^{*}=H_{\mathfrak{p}} \perp \mathfrak{O}_{\mathfrak{p}} \pi^{-e} w$ where $e=\operatorname{ord}_{p} 2$. There are now two new invariant lattices

$$
E_{\mathfrak{p}}=\pi M_{\mathfrak{p}}^{*}+\mathfrak{D}_{\mathfrak{p}}\left(u+v+\pi^{-e} w\right)
$$

and its dual $E_{\mathrm{p}}^{\sharp}$. It can be easily verified using the generators in Theorem 4.2 that $E_{\mathfrak{p}}$ is a $S U\left(M_{\mathfrak{p}}\right)$-invariant lattice; it follows that the dual $E_{p}^{\#}$ is also invariant.

Let $N_{p}$ be a primitive invariant sublattice of $M_{p}^{*}$. As in the proof of 2.4, there exists an element $x=\alpha u+v+\beta w$ in $N_{\mathfrak{p}}$ with $\alpha$ and $\pi^{e} \beta$ in $\mathfrak{O}_{p}$. Since $\pi=\zeta, T_{\pi}(u)(x)$ is in $N_{p}$. Hence $\pi M_{p^{*}} \subseteq N_{p}$. Assume first that $\pi^{e} \beta$ is a unit. Then $\pi x \in N_{p}$ forces $\pi^{1-e} w \in N_{p}$ and $\pi M_{p}^{*} \subseteq N_{p}$. If $\alpha$ is not a unit, then the image of $v+\pi^{-e} w$ under $E\left(v, \pi^{e} w\right)$ is in $N_{p}$. Hence $v \in N_{\mathfrak{p}}$ and $M_{p^{*}} \subseteq N_{p}$. Assume, therefore, $\alpha \equiv 1 \bmod \pi$. We have now shown, when $\pi^{e} \beta$ is a unit, $E_{\mathrm{p}} \subseteq N_{\text {p }}$. Moreover, $E_{\mathfrak{p}} \neq N_{p}$ forces $M_{p^{*}} \subseteq N_{p}$. Now assume $\pi^{e} \beta$ is not a unit and apply $E\left(u, \pi^{e} w\right)$ to $x$. This gives $u+\pi^{e} w$ is in $N_{p}$. The isometry $u \mapsto v, v \mapsto u, w \mapsto-w$ is in $S U\left(M_{p}\right)$. Hence both $v-\pi^{e} w$ and $u+v$ are in $N_{p}$. Define

$$
G_{\mathfrak{p}}=\pi M_{\mathfrak{p}^{*}}+\mathfrak{D}_{\mathfrak{p}}(u+v)+\mathfrak{O}_{\mathfrak{p}}\left(v+\pi^{e} w\right) .
$$

Then $\pi^{-1} G_{p}=E_{p}^{*}$, the dual lattice of $E_{p}$. Now, if $\pi^{e} \beta$ is not a unit, $G_{\mathfrak{p}} \subseteq N_{\mathfrak{p}}$ and if $G_{\mathfrak{p}} \neq N_{\mathfrak{p}}$, necessarily $M_{p^{*}} \subseteq N_{\mathfrak{p}}$. In summary,

5.1. The only exceptional three dimensional invariant lattices are of the form $\mathfrak{a}_{\mathfrak{p}} E_{\mathfrak{p}}$ and $\mathfrak{a}_{\mathfrak{p}} E_{\mathfrak{p}}^{*}$, with $\mathfrak{a}_{\mathfrak{p}}$ a fractional ideal in $K_{p}$.

Now consider the more complicated situation when $\operatorname{dim} V=4$ and $M_{\mathfrak{p}}=H_{\mathfrak{p}} \perp B_{\mathfrak{p}}$ with $w, z$ a basis of $B_{\mathfrak{p}}$ having $\Phi(w, z)=1$ and $z \in M_{p^{*}}$. Let $f$ be the minimal integer such that $\pi^{f} w$ is in $M_{p^{*}}$. Then

$$
M_{\mathfrak{p}^{*}}=H_{\mathfrak{p}} \perp\left(\mathfrak{Q}_{\mathfrak{p}} \pi^{f} w+\mathfrak{Q}_{\mathfrak{p}} z\right) .
$$

If $f=0$, then $M_{\mathfrak{p}^{*}}=M_{\mathfrak{p}}$ and it is easily verified that $M_{\mathfrak{p}}$ is the only primitive invariant lattice. Assume, therefore, $1 \leqq f \leqq e$. Now $z$ can be chosen with $q(z)$ in $p 0_{p}$. For $1 \leqq g \leqq f$, define

$$
E(g)_{\mathfrak{p}}=\pi M_{\mathfrak{p}^{*}}+\mathfrak{D}_{\mathfrak{p}} \pi^{g} w+\mathfrak{D}_{\mathfrak{p}}\left(u+v+\pi^{-f} z\right)
$$

and

$$
G(g)_{\mathfrak{p}}=\pi M_{p^{*}}+\mathfrak{O}_{\mathfrak{p}}(u+v)+\mathfrak{S}_{\mathfrak{p}} \pi^{1-g} z+\mathfrak{D}_{\mathfrak{p}}\left(u+\pi^{f} w\right) .
$$

Then $G(g)_{\mathfrak{p}}=\pi^{-1} E(g)_{\mathfrak{p}}^{*}$ and using Theorem 4.2 we can check that these 
lattices are all $S U\left(M_{\mathfrak{p}}\right)$-invariant. However, except when $f=1$, these are not the only new invariant lattices that arise. We shall only consider $f=1$ in detail; this includes the case where 2 is prime in $F_{p}$.

Let $N_{\mathfrak{p}}$ be a primitive $S U\left(M_{\mathfrak{p}}\right)$-invariant sublattice of $M_{\mathfrak{p}}^{*}$. Again $N_{\mathrm{p}}$ contains an element $x=\alpha u+v+\beta w+\gamma z$ with $\alpha, \beta$ and $\pi^{f} \gamma$ in $\mathfrak{\Omega}_{p}$. Applying $T_{\pi}(u)$ to $x$ gives $\pi u \in N_{\mathfrak{p}}$ and hence $\pi M_{p^{*}} \subseteq N_{\mathfrak{p}}$. Since $E(u, z)(x)$ is in $N_{\mathfrak{p}}$, we can conclude that $\beta$ is in $\pi \mathfrak{N}_{\mathfrak{p}}$ and $z$ is in $N_{\mathfrak{p}}$, for otherwise $M_{p^{*}} \subseteq N_{p}$. Assume first that $\gamma$ is in $\pi^{1-f} \mathfrak{\Omega}_{p}$. Then $E\left(u, \pi^{f} w\right)(x) \in N_{p}$ gives $u+\pi^{f} w$ and $u+v$ are both in $N_{p}$. Hence $G(1)_{\mathfrak{p}} \subseteq N_{\mathfrak{p}}$. If $f=1$ and $G(1)_{\mathfrak{p}} \neq N_{\mathfrak{p}}$, necessarily $M_{p^{*}} \cong N_{\mathfrak{p}}$. Now

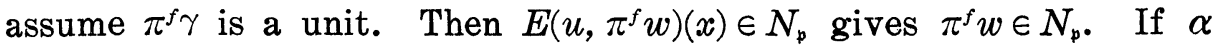
is a nonunit, applying $E\left(v, \pi^{f} w\right)$ to $x$ leads to $M_{p^{*}} \subseteq N_{p}$. Hence $\alpha \equiv$ $1 \bmod \pi$ and now $u+v+\beta w+\pi^{-f} z$ is in $N_{p}$ with $\beta \in \pi \mathfrak{O}_{p}$. Again, if $f=1$, this gives $E(1)_{\mathfrak{p}} \subseteq N_{\mathfrak{p}}$ and, if $E(1)_{\mathfrak{p}} \neq N_{\mathfrak{p}}$, necessarily $M_{\mathfrak{p}^{*}} \subseteq$ $N_{p}$. Hence,

5.2. For $f=1$ the only exceptional four dimensional invariant lattices are of the form $\mathfrak{a}_{\mathfrak{p}} E(1)_{\mathfrak{p}}$ and $\mathfrak{a}_{\mathfrak{p}} E(1)_{\mathfrak{p}}^{\sharp}$, with $\mathfrak{a}_{\mathfrak{p}}$ a fractional ideal in $K_{p}$.

For $f \geqq 2$, the analysis of the exceptional lattices is more complicated, but could be carried out in the above manner.

6. Global results. We start by proving Theorem A; in fact, this result remains valid even if $M$ is not unimodular.

First let $N$ be a $S U(M)$-invariant sublattice of $M$. We must prove $N_{\mathfrak{p}}=\mathfrak{O}_{\mathfrak{p}} N$ is $S U\left(M_{\mathfrak{p}}\right)$-invariant at all finite prime spots $\mathfrak{p}$ of $F$. Fix a finite prime spot $q$ and an isometry $\psi_{q}$ in $S U\left(M_{q}\right)$. By the approximation theorem of Shimura [8; 5.12], there exists a $\varphi$ in $S U(V)$ with local extension $\varphi_{q}$ close to $\psi_{q}$ at the spot $q$ and $\varphi_{p}\left(M_{p}\right)=$ $M_{\mathfrak{p}}$ elsewhere. Since $\psi_{q}\left(M_{q}\right)=M_{q}$, we have $\varphi_{q}\left(M_{q}\right)=M_{q}$ if $\varphi_{q}$ is sufficiently close to $\psi_{\mathrm{a}}$ and hence $\varphi(M)=M$. Thus $\varphi$ is in $S U(M)$ and hence $\varphi(N)=N$. Therefore, $\varphi_{\mathrm{q}}\left(N_{\mathrm{q}}\right)=N_{\mathrm{q}}$ and if $\varphi_{\mathrm{q}}$ is sufficiently close to $\psi_{q}$, necessarily $N_{q}$ is invariant under $\psi_{q}$.

Conversely, let $N$ be a lattice in $M$ with $N_{\mathfrak{p}}=\mathfrak{\Omega}_{p} N$ a $S U\left(M_{\mathfrak{p}}\right)$ invariant lattice at all finite prime spots $\mathfrak{p}$. We must prove $\varphi(N)=N$ for all $\phi$ in $S U(M)$. Clearly, however, $\phi_{\mathfrak{p}} \in S U\left(M_{\mathfrak{p}}\right)$ so that $\varphi(N)_{\mathfrak{p}}=$ $\varphi_{p}\left(N_{p}\right)=N_{p}$. The result now follows as in O'Meara [7; $\S 81 \mathrm{E}$ ]. Notice that this half of the proof does not require that $\Phi$ be indefinite. This completes the proof of Theorem A.

We can also construct global invariant lattices from local ones as follows.

Proposition 6.1. At each finite spot $\mathfrak{p}$ of $F$ assume given a 
$S U\left(M_{\mathfrak{p}}\right)$-invariant sublattice $J_{\mathfrak{p}}$ of $M_{\mathfrak{p}}$ with $J_{\mathfrak{p}}=M_{\mathfrak{p}}$ almost always. Then there exists a sublattice $N$ of $M$ such that for each spot $\mathfrak{p}$

$$
N_{\mathfrak{p}}=\mathfrak{D}_{\mathfrak{p}} N=J_{\mathfrak{p}} .
$$

Proof. This is an immediate consequence of $[2 ; 2.4]$.

We conclude this paper by giving more explicitly the invariant lattices when $F$ is the rational field $\boldsymbol{Q}$. Now $K=\boldsymbol{Q}(\sqrt{m})$ with $m$ a square free integer. Let $p$ be a rational prime. Then $p$ splits in $K$ if either $p=2$ and $m \equiv 1 \bmod 8$, or $p$ is odd and $(m / p)=1$. Otherwise, for $p=2$, we have an unramified extension if $m \equiv$ $5 \bmod 8$, a ramified unit extension with $h=0$ if $m \equiv 3 \bmod 4$, and a ramified prime extension if $m$ is even.

Let $M$ be a unimodular lattice on an indefinite hermitian space $V$ over $\boldsymbol{Q}(\sqrt{m})$. Except when $\boldsymbol{Q}_{2}(\sqrt{m})$ is a ramified extension of $\boldsymbol{Q}_{2}$, the only primitive invariant sublattice is $M_{p}$. Hence, when $m \equiv 1 \bmod 4$, the $S U(M)$-invariant lattices are the $a M$ with $a$ a fractional ideal in $\boldsymbol{Q}(\sqrt{m})$.

When $m \equiv 3 \bmod 4$ or $m$ is even, $\boldsymbol{Q}_{2}(\sqrt{m})$ is a ramified extension of $\boldsymbol{Q}_{2}$ and $M_{2}$ can support other local invariant lattices. If the rank of $M$ is odd, the invariant lattices are the $a N$ with a a fractional ideal and $N_{2}$ one of the lattices $M_{2^{*}}, M_{2}$ or $M_{2}^{*}$, together with $E_{2}$ and $E_{2}^{\#}$ when $m$ is even and $\operatorname{dim} V=3$.

Finally, when the rank of $M$ is even there are a number of possibilities. If $\Phi$ is an even form, namely if $M_{2^{*}}=M_{2}$, the only invariant sublattices are the $a M$ with $a$ a fractional ideal. If $\Phi$ is an odd form and $m \equiv 3 \bmod 4$ or $m$ is even, there are five lattices $N_{2}$ lying between $M_{2^{*}}$ and $M_{2}^{*}$. If $M_{2}=H_{2} \perp J_{2} \perp\left(\mathfrak{D}_{2} w+\mathfrak{\Omega}_{2} z\right)$ with $\Phi(w, z)=1,2 q(w)$ a unit and $q(z) \in \mathfrak{D}_{\mathfrak{p}}$, these five lattices are $M_{2}$, $M_{2^{*}}, M_{2}^{*}$,

$$
H_{2} \perp J_{2} \perp\left(\mathfrak{D}_{2} \pi w+\mathfrak{D}_{2} \pi^{-1} \boldsymbol{z}\right)
$$

and

$$
H_{2} \perp J_{2} \perp\left(\mathfrak{D}_{2} \pi w+\mathfrak{O}_{2}\left(w+\pi^{-1} z\right)\right) .
$$

For $\operatorname{dim} V \geqq 6$ and for $\operatorname{dim} V=4$ when $m \equiv 3 \bmod 4$, the invariant lattices are the $\mathfrak{a} N$ with $\mathfrak{a}$ a fractional ideal, $N_{2}$ one of these five lattices and $N_{p}=M_{p}$ for $p$ odd. When $\operatorname{dim} V=4$ and $m$ is even, $N_{2}$ can also be one of the dual pair of exceptional lattices $E(1)_{2}$ and $E(1)_{2}^{\#}$ obtained in the previous section.

\section{REFERENCES}

1. R. Baeza, Eine Zerlegung der unitären Gruppe über lokalen Ringen, Arch. Math. (Basel), 24 (1973), 144-157. 
2. L. J. Gerstein, Integral decomposition of hermitian forms, Amer. J. Math., 92 (1970), 398-418.

3. K. Hayakawa, Generation of local integral unitary groups over an unramified dyadic local field, J. Fac. Sci., Univ. Tokyo, Sect. I, 15 (1968), 1-11.

4. R. Jacobowitz, Hermitian forms over local fields, Amer. J. Math., 84 (1962), 441-465.

5. D. G. James, Orthogonal groups of dyadic unimodular quadratic forms II, Pacific J. Math., 52 (1974), 425-441.

6. - On the normal subgroups of integral orthogonal groups, Pacific J. Math., 52 (1974), 107-114.

7. O. T. O'Meara, Introduction to Quadratic Forms, Springer-Verlag, Berlin, 1963.

8. G. Shimura, Arithmetic of unitary groups, Ann. of Math., 79 (1964), 369-409.

Received December 21, 1976 and in revised form April 29, 1977. This research was partially supported by the National Science Foundation.

The Pennsylvania State University

UNIVERSITY PARK, PA 16802 


\title{
PACIFIC JOURNAL OF MATHEMATICS
}

\section{EDITORS}

\author{
RICHARD ARENS (Managing Editor) \\ University of California \\ Los Angeles, CA 90024 \\ Charles W. Curtis \\ University of Oregon \\ Eugene, OR 97403 \\ C. C. MOORE \\ University of California \\ Berkeley, CA 94720
}

\section{J. DUGUNDJI}

Department of Mathematics

University of Southern California

Los Angeles, CA 90007

R. FINN and J. MILGRAM

Stanford University

Stanford, CA 94305

\section{ASSOCIATE EDITORS}
E. F. BECKENBACH
B. H. NeumanN
F. WOLF
K. YoSHIDA

\section{SUPPORTING INSTITUTIONS}

UNIVERSITY OF BRITISH COLUMBIA

CALIFORNIA INSTITUTE OF TECHNOLOGY

UNIVERSITY OF CALIFORNIA

MONTANA STATE UNIVERSITY

UNIVERSITY OF NEVADA, RENO

NEW MEXICO STATE UNIVERSITY

OREGON STATE UNIVERSITY

UNIVERSITY OF OREGON

OSAKA UNIVERSITY

\author{
UNIVERSITY OF SOUTHERN CALIFORNIA \\ STANFORD UNIVERSITY \\ UNIVERSITY OF HAWAII \\ UNIVERSITY OF TOKYO \\ UNIVERSITY OF UTAH \\ WASHINGTON STATE UNIVERSITY \\ UNIVERSITY OF WASHINGTON \\ $\stackrel{*}{*} \stackrel{*}{*} \stackrel{*}{*}$ AMERICAN MATHEMATICAL SOCIETY
}

The Supporting Institutions listed above contribute to the cost of publication of this Journal, but they are not owners or publishers and have no responsibility for its content or policies.

Mathematical papers intended for publication in the Pacific Jaurnal of Mathematics should be in typed form or offset-reproduced, (not dittoed), double spaced with large margins. Please do not use built up fractions in the text of your manuscript. You may however, use them in the displayed equations. Underline Greek letters in red, German in green, and script in blue. The first paragraph or two must be capable of being used separately as a synopsis of the entire paper. Items of the bibliography should not be cited there unless absolutely necessary, in which case they must be identified by author and Journal, rather than by item number. Manuscripts, in triplicate, may be sent to any one of the editors. Please classify according to the scheme of Math. Reviews, Index to Vol. 39. All other communications should be addressed to the managing editor, or Elaine Barth, University of California, Los Angeles, California, 90024.

The Pacific Journal of Mathematics expects the author's institution to pay page charges, and reserves the right to delay publication for nonpayment of charges in case of financial emergency.

100 reprints are provided free for each article, only if page charges have been substantially paid. Additional copies may be obtained at cost in multiples of 50 .

The Pacific Journal of Mathematics is issued monthly as of January 1966. Regular subscription rate: $\$ 7200$ a year (6 Vols., 12 issues). Special rate: $\$ 36.00$ a year to individual members of supporting institutions.

Subscriptions, orders for back numbers, and changes of address should be sent to Pacific Journal of Mathematics, 103 Highland Boulevard, Berkeley, California, 94708.

PUBLISHED BY PACIFIC JOURNAL OF MATHEMATICS, A NON-PROFIT CORPORATION

Printed at Kokusai Bunken Insatsusha (International Academic Printing Co., Ltd.). 8-8, 3-chome, Takadanobaba, Shinjuku-ku, Tokyo 160, Japan.

Copyright (C) 1975 by Pacific Journal of Mathematics Manufactured and first issued in Japan 


\section{Pacific Journal of Mathematics}

Vol. 72, No. 2

February, 1977

George E. Andrews, Plane partitions. II. The equivalence of the

Bender-Knuth and MacMahon conjectures ................. 283

Lee Wilson Badger, An Ehrenfeucht game for the multivariable quantifiers

of Malitz and some applications ......................... 293

Wayne C. Bell, A decomposition of additive set functions ............ 305

Bruce Blackadar, Infinite tensor products of $C^{*}$-algebras ............. 313

Arne Brøndsted, The inner aperture of a convex set .............. 335

N. Burgoyne, Finite groups with Chevalley-type components........... 341

Richard Dowell Byrd, Justin Thomas Lloyd and Roberto A. Mena, On the retractability of some one-relator groups .....................

Paul Robert Chernoff, Schrödinger and Dirac operators with singular potentials and hyperbolic equations .................... 361

John J. F. Fournier, Sharpness in Young's inequality for convolution ....... 383

Stanley Phillip Franklin and Barbara V. Smith Thomas, On the metrizability

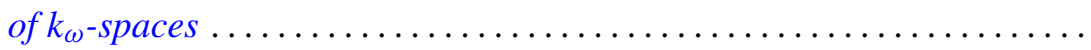

David Andrew Gay, Andrew McDaniel and William Yslas Vélez, Partially normal radical extensions of the rationals .................... 403

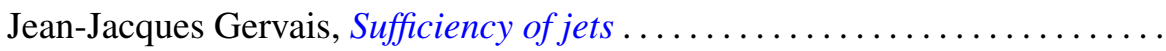

Kenneth R. Goodearl, Completions of regular rings. II . .

Sarah J. Gottlieb, Algebraic automorphisms of algebraic groups with stable maximal tori

Donald Gordon James, Invariant submodules of unimodular Hermitian forms.....

J. Kyle, $W_{\delta}(T)$ is convex.

Ernest A. Michael and Mary Ellen Rudin, A note on Eberlein compacts ...

Ernest A. Michael and Mary Ellen Rudin, Another note on Eberlein compacts ....

Thomas Bourque Muenzenberger and Raymond Earl Smithson, Fixed point theorems for acyclic and dendritic spaces.

Budh Singh Nashier and A. R. Rajwade, Determination of a unique solution of the quadratic partition for primes $p \equiv 1(\bmod 7)$.

Frederick J. Scott, New partial asymptotic stability results for nonlinear ordinary differential equations ....................

Frank Servedio, Affine open orbits, reductive isotropy groups, and dominant gradient morphisms; a theorem of Mikio Sato..........

D. Suryanarayana, On the distribution of some generalized square-full integers.................................. 